\title{
Clinical Characteristics and Prognostic Analysis of Gynecologic Cancer with Spinal Metastases: A Single-Center Retrospective Study
}

This article was published in the following Dove Press journal: Cancer Management and Research

\author{
Shuzhong Liu',* \\ Xi Zhoul,* \\ An Song ${ }^{2}$ \\ Zhen $\mathrm{Huo}^{3}$ \\ Siyuan Yao' \\ Yipeng Wang' \\ Yong Liu (iD) ${ }^{1, *}$ \\ Ling-Ya $\operatorname{Pan}^{4, *}$
}

'Department of Orthopaedic Surgery, Peking Union Medical College Hospital, Peking Union Medical College and Chinese Academy of Medical Sciences, Beijing, People's Republic of China; ${ }^{2}$ Department of Endocrinology, Key Laboratory of Endocrinology, National Health and Family Planning Commission, Peking Union Medical College Hospital, Chinese Academy of Medical Sciences \& Peking Union Medical College, Beijing, People's Republic of China; ${ }^{3}$ Department of Pathology, Peking Union Medical College Hospital, Chinese Academy of Medical

Sciences \& Peking Union Medical College, Beijing, People's Republic of China;

${ }^{4}$ Department of Obstetrics and

Gynecology, Peking Union Medical College Hospital, Chinese Academy of Medical Sciences \& Peking Union Medical College, Beijing 100730, People's Republic of China

*These authors contributed equally to this work

Correspondence: Yong Liu Department of Orthopaedic Surgery, Peking Union Medical College Hospital, Peking Union Medical College and Chinese Academy of Medical Sciences, No. I Shuaifuyuan Wangfujing, Beijing 100730 , People's Republic of China,

Email liuyong_pumch@I63.com

Ling-Ya Pan

Department of Obstetrics and Gynecology, Peking Union Medical College Hospital, Chinese Academy of Medical Sciences \& Peking Union Medical College, Beijing 100730, People's Republic of China

Email panly@pumch.cn
Objective: The purpose of this study is to provide key information on the clinical characteristics, surgical treatment, and potential prognostic factors in patients with metastatic spinal gynecologic cancer (MSGC), with a view to their application in clinical practice.

Methods: From January 2010 to January 2020, we performed a retrospective analysis of 14 patients with MSGC who underwent surgical treatment in a single center. Surgical treatment was performed on 14 patients, and a total of 14 operations were performed. The survival time of patients after spinal surgery was analyzed by Kaplan-Meier and Cox regression analysis. Results: The average age of patients was 51.9 years (range 25-70). The average time from initial surgery to the discovery of spinal metastasis was 60.3 months $(2-180)$, and the average follow-up time was 19.2 months (2-55). Spinal tumor progression was found in 9 patients, and 12 patients $(85.7 \%)$ died during follow-up. In univariate analysis, extraosseous visceral metastasis $(\mathrm{p}=0.024)$, revised Tokuhashi stage $(\mathrm{p}=0.025)$, Tomita stage $(\mathrm{p}=$ $0.005)$, and number of spinal lesions $(\mathrm{p}=0.038)$ were associated with overall survival (OS). Extraosseous visceral metastasis $(p=0.026)$, revised Tokuhashi stage $(p=0.014)$, Tomita stage $(\mathrm{p}=0.001)$, and gynecological cancer type $(\mathrm{p}=0.039)$ were associated with progression-free survival.

Conclusion: Surgical treatment is an effective treatment for MSGC and relieves pain, restores function and rebuilds stability. Based on our single-center experience, extraosseous visceral metastasis, revised Tokuhashi stage, Tomita stage, and gynecological cancer type may be potential prognostic factors for OS.

Keywords: clinical prognosis, gynecological cancer, overall survival, progression-free survival, spinal metastasis, surgical treatment

\section{Introduction}

Gynecological cancers (GC) are common diseases of the female reproductive system. ${ }^{1}$ The overall incidence rate is second only to breast cancer in women, which seriously threatens women's lives and physical and mental health. ${ }^{1,2}$ In recent years, with more awareness around gynecological diseases and standardized treatment of tumors, the morbidity and mortality of gynecological tumors has declined. ${ }^{1-3}$ However, some patients still relapse or develop metastases within 23 years after treatment. ${ }^{4,5}$ Many patients develop pelvic or distant metastases during or after treatment, which can be metastatic to bones, liver, brain, lungs or kidneys. In China, GC accounts for about $12 \%$ of all cancer types in women. ${ }^{1}$ The most common types are cervical cancer, endometrial cancer, and ovarian cancer, with 
98,900, 63,400, and 52,100 newly diagnosed cases each year, respectively, and in total, 30,500, 21,800 and 22,500 patients, respectively, die from these diseases. ${ }^{1,2}$ When spinal metastasis occurs, patients often suffer from unbearable pain, pathological fractures, or the serious complications of quadriplegia, which seriously affect quality of life and survival. However, awareness of MSGC in practice is low and patients often do not receive adequate treatment.

The spine is the most common site of bone metastasis. According to reports, the incidence of clinical bone metastases in cervical cancer is about $1.1-5.2 \%$, and spinal metastases account for $46.7-59.0 \%$ of all bone metastases. ${ }^{6,7}$ Endometrial cancer has a bone metastasis rate of about $0.3-1.8 \%$, and a spinal metastasis rate of $36.2-44.8 \% .{ }^{8,9}$ The rate of bone metastases from other GCs (eg, ovarian cancer, fallopian tube cancer) is relatively low $(<1 \%){ }^{10}$

In recent years, the crucial role of surgery in the treatment of spinal metastatic tumors has been gradually recognized and widely accepted. However, because it is often overlooked in clinical practice, the clinical diagnosis and optimal treatment of MSGC is yet to be defined. Liu et al reported 6 cases of patients with MSGC and emphasized the importance of spinal surgery. ${ }^{11}$ Gao et al also reviewed 28 consecutive patients with MSGC, 28 of whom underwent surgical treatment, and identified potential factors associated with spinal metastasis and the prognostic factors influencing OS. ${ }^{3}$ However, the surgical methods reported in the study were varied, which may affect the reliability of the authors' conclusions therefore further studies with larger sample sizes are needed. Here, we report the clinical characteristics and comprehensive diagnosis and treatment experience of 14 patients with MSGC who were diagnosed and treated in a single center for 10 years. Our study includes one of the largest sample size reported so far and is designed to improve clinical awareness.

\section{Materials and Methods}

This was a retrospective analysis of 14 consecutive MSGC patients who underwent spinal surgery at our orthopedic oncology center from January 2010 to January 2020. The study was approved by the hospital ethics committee and informed consent was obtained from all patients.

All 14 patients had pathologically confirmed gynecological cancer and underwent surgical resection or biopsy of the primary tumor before admission. Diagnosis of
MSGC was confirmed based on the following three criteria: (1) imaging findings consistent with MSGC based on standard X-ray, computed tomography (CT), magnetic resonance imaging (MRI), bone scan or fluorodeoxyglucose (FDG) positron emission tomography-computed tomography (PET/CT); (2) a history of major gynecological cancer; (3) diagnosis confirmed by biopsy or open surgery pathological specimens. Preoperative neurological status was classified according to Frankel score and American Spinal Injury Association (ASIA) injury classification. The quality of life of all patients was evaluated using the Karnofsky score, Eastern Cooperative Oncology Group (ECOG) score, and visual analogue scale (VAS) scoring. Surgery was performed by the team led by Professor Liu. The spinal instability neoplastic score (SINS) system was used to assess the stability of the spine, and the revised Tokuhashi and Tomita scoring systems were used to assess prognosis and assist in the development of surgical procedures. The two researchers conducted a detailed review and summary of patients' clinical and surgical records, clinical data, imaging data and pathology reports. PET-CT was performed to identify possible metastatic sites. Frankel score, VAS and ECOG score were used to assess neurological status, pain level and performance status, respectively. Retrospective analysis was performed taking into account basic clinical data, spinal metastasis site, Frankel score, Karnofsky performance score (KPS), SINS, VAS, revised Tokuhashi score, Tomita score, urinary and fecal conditions, spinal pathological fractures, primary tumors, extraspinal spinal metastases, visceral metastases, and bone damage (osteolytic, osteogenic or mixed).

Surgical indications included: 1) initially defined spinal metastases with unbearable pain or neurological deficits; 2) disease control not achieved by conservative treatment; 3 ) no clear surgical contraindication after comprehensive evaluation by subspecialties; 4) the opinions of the obstetrics and gynecology department taken into account; 5) patients undergoing surgery provided informed consent. According to the location and scope of spinal tumors, individualized surgical strategies should be adopted for each patient. Adjunctive treatment recommendations were also needed postoperatively according to the individual condition of the patient.

Radiological assessments (spine X-ray, CT or MRI) were performed regularly at 3 and 6 months after surgery, followed by periodic assessments every 6 months for the next 2 years, and then periodically every year. For patients 
showing tumor progression, PET-CT was recommended for detailed evaluation. Follow-up data were collected during outpatient visits and telephone interviews. During follow-up, neurological function and improvement quality were reassessed according to the VAS score, Frankel and Karnofsky scoring systems. The follow-up period was defined as the interval from the date of spinal surgery to the date of death, or until the last follow-up.

\section{Statistical Analysis}

PFS was defined as the time period between the date of spinal surgery for spinal metastasis and the date of recurrence or progression of spinal metastases observed by imaging examination. OS was defined from first day of imaging until the patient's death due to disease or spinal metastasis until the end of May 2020. Quantitative data are expressed in terms of counts and percentages using mean or median (range) and qualitative description data. The Kaplan-Meier method was used to evaluate the survival rate, and the Log rank test was used to determine the difference. $\mathrm{P}<0.05$ was considered statistically significant. All statistical calculations were performed using SPSS Statistics version 23.0 (IBM corp., New York, USA).

\section{Results}

\section{Patient Characteristics}

The clinical data of all patients are listed in Table 1 . The average age was 51.9 years (range 25-70), and all patients underwent spinal surgery. The average time from the initial surgery to the discovery of spinal metastasis was 60.3 months (range 2-180). Common symptoms in patients with spinal metastases included progressively intractable or persistent pain, muscle weakness, numbness of the limbs, and even paraplegia or diarrhea. The preoperative duration of symptoms was 2.8 months (range 0.3-6).

All 14 patients underwent surgical resection of the primary lesion. Postoperative pathology confirmed that the main tumor types included 7 cases of cervical cancer $(50.0 \%), 4$ cases of endometrial cancer $(28.6 \%)$, and 2 cases of ovarian cancer $(14.3 \%)$, and 1 case of fallopian tube carcinoma (7.1\%). Of all 14 patients, 10 had unsynchronized metastasis (spine metastasis occurred after diagnosis of GC). Before admission, all patients received neoadjuvant chemotherapy, and 9 received radiation therapy.

\section{Characteristics of Spinal Metastases}

In our case series, the lesions were mainly located in the thoracic spine ( 8 cases), lumbar spine ( 8 cases) and sacrum ( 2 cases), with 10 cases $(71.4 \%$ ) and 4 cases $(28.6 \%)$ suffering from single focus and multiple focus spinal metastatic disease, respectively. The largest disease burden was seen in one patient with 6 spinal segments involved.

Overall, 13 cases showed osteolytic changes and one patient showed osteogenic changes. Eight patients had vertebral compression fractures (Figure 1) and spinal MRI showed that $42.9 \%(6 / 14)$ of patients had spinal epidural compression, and 42.9\% (6/14) had spinal lesions with paravertebral expansion. Six (42.9\%) patients had uncontrolled visceral metastases involving the brain, liver, and lung, before spinal surgery.

\section{Treatment and Follow-Up}

Before spinal surgery, 11 patients $(80.0 \%)$ had normal neurological function (Frankel grade $\mathrm{E}$ ). Two patients (14.3\%) were Frankel grade C, and one patient $(7.1 \%)$ was Frankel grade A. Therefore, 3 patients $(21.4 \%)$ were deemed to have neurological defects before surgery. Surgical treatment was performed by the team of Professor Liu in our department, after at least 5 years of professional training. A total of 14 operations were performed, including eight patients who underwent posterior tumor resection, spinal cord decompression, and titanium rod system spinal reconstruction (Figure 2). The average blood loss during operation was $650 \mathrm{~mL}$ (range 100-1200). Six patients underwent minimally invasive bone cement percutaneous vertebroplasty (Figure 3), with an average blood loss of $35 \mathrm{~mL}$ (range 20-30) during the operation. No obvious complications occurred during the perioperative period, and the original treatment plan continued after the operation in all cases. All 14 patients received bisphosphonate treatment after surgery. The results of postoperative immunohistochemistry supported the pathological diagnosis of MSGC. For isolated spinal metastatic lesions, we prefer total resection. Eight patients in this study underwent open surgery to maximize the removal of spinal metastatic disease, supplemented with postoperative adjuvant therapy. Six patients underwent percutaneous bone cement reconstruction to maximize the stability of the spine, supplemented with postoperative adjuvant therapy. During the 3-month follow-up, the neurological status of 3 patients with nerve injury showed a Frankel score improvement 


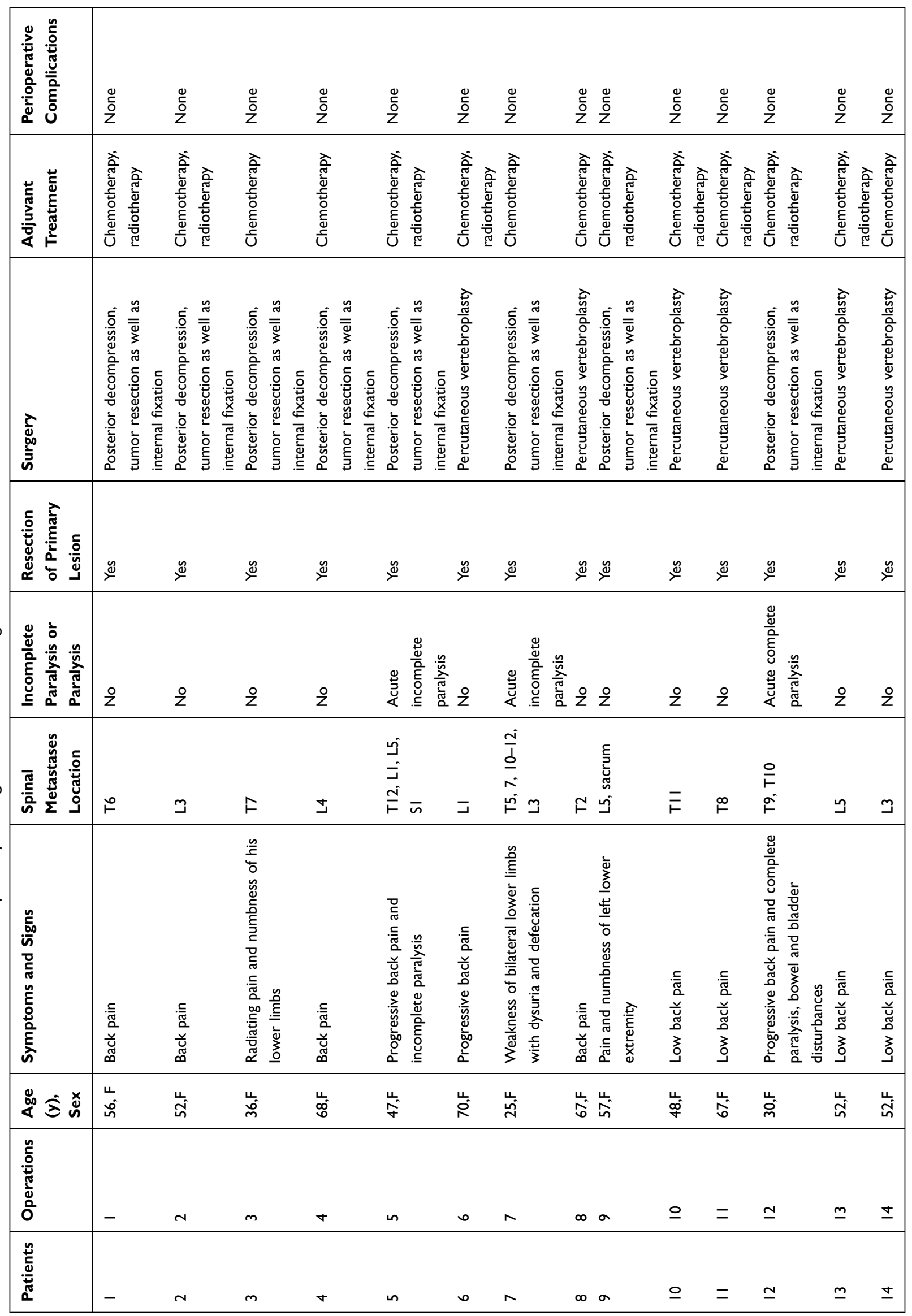



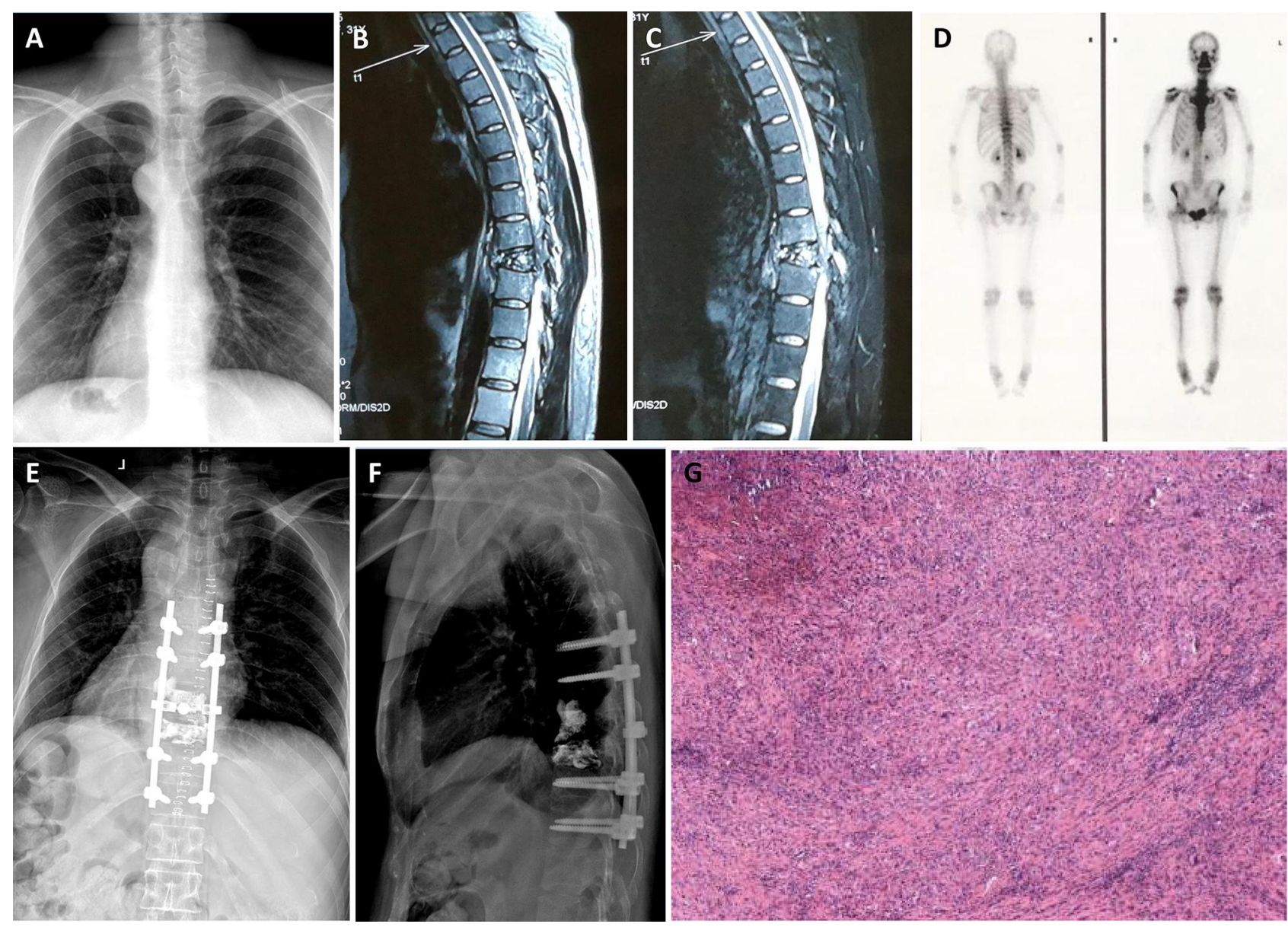

Figure I Radiographic and pathological images of a representative 30-year-old female patient (Case \#I2). (A) Preoperative X-ray. (B, C) Preoperative sagittal MRI scan revealing vertebral fracture caused by spinal metastases. (D) Bone scan revealing metastasis of the spine. (E, F) Postoperative $X$-rays of the thoracic spine. (G) Microphotography showing significant nuclear pleomorphism with prominent nucleoli (H\&E, original magnification I00x).

of 1-2 grades. In our series, the most common histological subtypes were cervical cancer $(\mathrm{n}=7,50.0 \%)$, endometrial cancer $(\mathrm{n}=4,28.6 \%)$, ovarian cancer $(\mathrm{n}=2$, $14.3 \%)$, and fallopian tube carcinoma $(\mathrm{n}=1,7.1 \%)$. The OS was 19.2 months (range 2-55). Fourteen patients underwent an average of 19.2 months (2-55) of postoperative follow-up. The 1-year and 2-year survival rate of all patients was $64.3 \%$ and $21.4 \%$, respectively.

\section{Univariate Analysis of Prognostic Factors}

According to Kaplan-Meier curve analysis (Figure 4) and the log rank test, extraosseous visceral metastasis $(\mathrm{p}=$ $0.024)$, revised Tokuhashi stage $(\mathrm{p}=0.025)$, Tomita stage $(\mathrm{p}=0.005)$, and number of spinal lesions $(\mathrm{p}=$ 0.038) were associated with OS (Figure 5). Extraosseous visceral metastasis $(\mathrm{p}=0.026)$, revised Tokuhashi stage $(\mathrm{p}$ $=0.014)$, Tomita stage $(\mathrm{p}=0.001)$, and gynecological cancer type $(p=0.039)$ were associated with PFS
(Figure 6). No significant differences were found among other factors.

\section{Discussion}

GC represents multiple tumor types with similar disease origin, clinical diagnosis, treatment, and prognosis. Ovarian cancer has the worst prognosis overall, with a 5-year survival rate of $44.6 \%$, compared with $67.9 \%$ for cervical cancer and $81.5 \%$ for endometrial cancer. ${ }^{11}$ The prognosis among patients with MSGC from different tumor types is similar. The literature reports that the survival time for patients with bone metastases from cervical cancer and endometrial cancer is 6-10 months and 10-15 months, respectively. ${ }^{8,9}$ Due to few relevant reports and clinical neglect, the clinical features and prognosis of MSGC are not clear. For malignant tumors, bone metastasis is often a sign of poor prognosis. The average survival time of patients with uterine malignant tumors is $4.8-$ 6.6 months after the development of bone metastases, and 


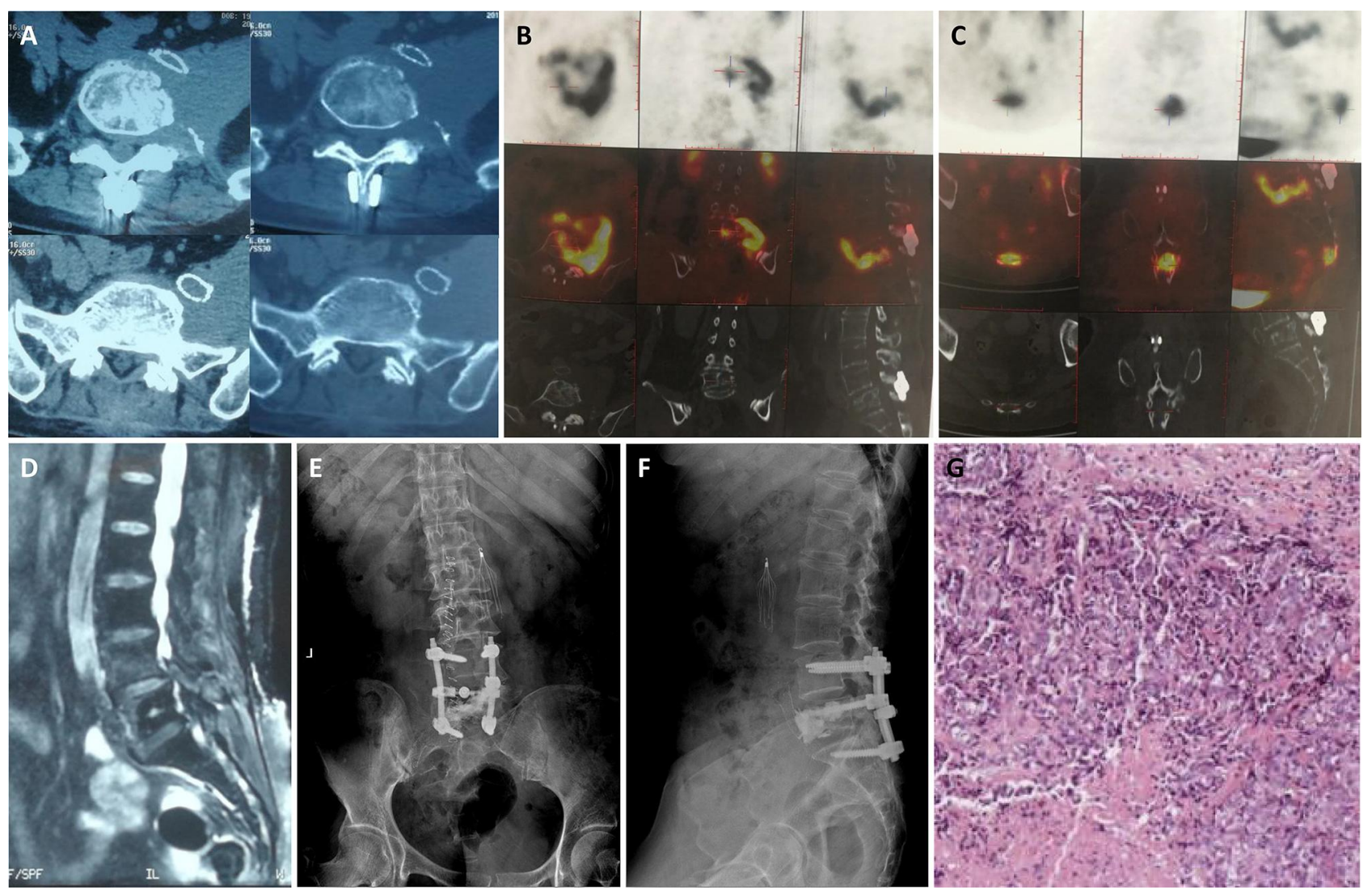

Figure 2 Radiographic and pathological images of a representative 57-year-old female patient (Case \#9). (A, D) Preoperative CT and MRI scan revealing vertebral metastases. (B, C) Positron emission tomography-computed tomography revealing metastases of the spine. (E, F) Postoperative $\mathbf{X}$-rays of the lumbar spine. (G) Microphotography showing significant nuclear pleomorphism with prominent nucleoli (H\&E, original magnification 100x).

the average time from diagnosis of the primary tumor to the death of patients with bone metastases is 10-12 months. ${ }^{12,13}$ Liu et al reported 6 cases of GC metastases to the spine including review of postoperative survival rates. ${ }^{11}$ The overall survival after spinal surgery was 27 months, and for cervical cancer and endometrial cancer was 32 months and 26 months, respectively. Rades et al reported that 7 patients with ovarian cancer and spinal metastases only received radiotherapy, and the survival time was 1-7 months (median 4 months). ${ }^{14}$ This study showed that the average survival time after spinal metastasis from gynecologic tumors was 19.2 months (2-55), and the average survival time from the diagnosis of the primary tumor to death was 60.3 months $(2-180)$.

Bone metastasis rarely occurs in cervical cancer, with an incidence of $0.8-2.3 \%{ }^{6,15,16}$ Among them, spinal metastasis is the most common $(36.36 \%) .{ }^{16}$ Bone metastases from ovarian malignant tumors are rare in the clinic, and reports show that the incidence of ovarian cancer bone metastases is only $0.1-0.6 \% .{ }^{17}$ Some studies have found that bone metastases in patients with cervical cancer and endometrial cancer generally occur within 2 years after the diagnosis of the primary tumor. ${ }^{15,16,18}$ Abdul-Karim et al reviewed 20 cases of cervical cancer and 17 cases of endometrial cancer and found that $71 \%$ of cervical cancer patients developed bone metastasis within 2 years after diagnosis; 4 patients with endometrium cancer developed bone metastasis within 6 months, and 2 patients developed bone metastasis within $2-5$ years. ${ }^{19}$

The following surgical indications should be strictly grasped: (1) severe symptoms or progressive neurological dysfunction; (2) intractable pain that is ineffective for conservative treatment; (3) pathological confirmation; (4) tumors that are insensitive to radiotherapy and chemotherapy; (5) spinal instability or collapse (6) patients whose expected survival is longer than three months. Due to the development of improved surgical techniques and equipment, surgical interventions have become more widely used in the clinical treatment of spinal metastases to reduce pain, restore and preserve nerve function, and enhance spinal stability. In our retrospective study, a total of 14 operations were performed. Eight patients underwent 

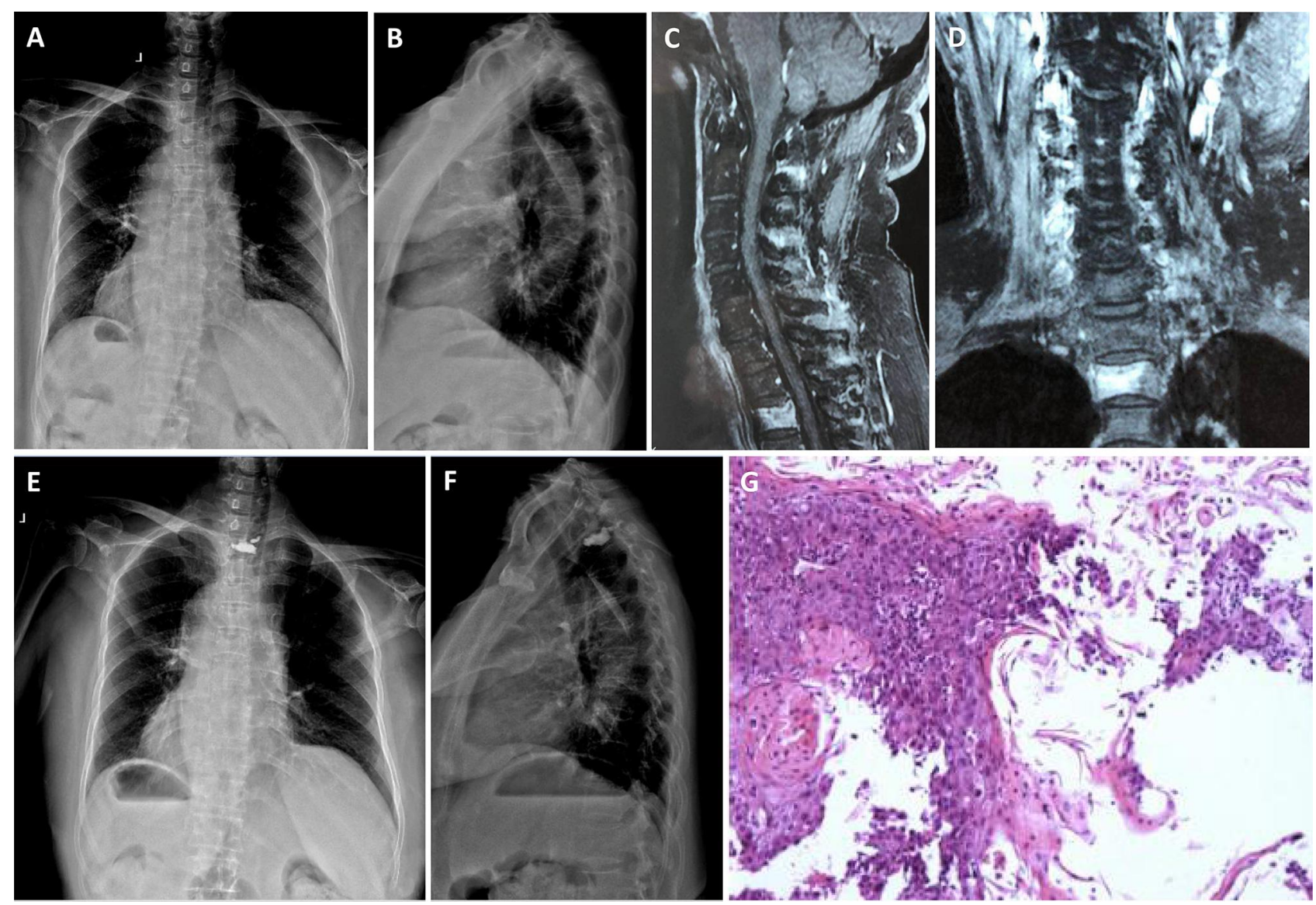

Figure 3 Radiographic and pathological images of a representative 67-year-old female patient (Case \#8). (A, B) Preoperative X-rays. (C, D) Preoperative MRI revealing vertebral metastases. (E, F) X-ray images of the thoracic spine obtained postoperatively. (G) Microphotography showing significant nuclear pleomorphism with prominent nucleoli (H\&E, original magnification 100x).

A

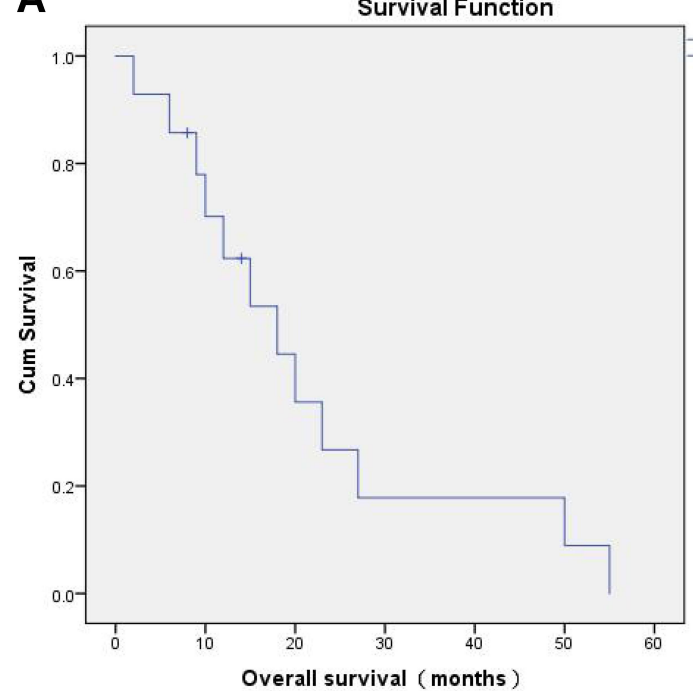

B

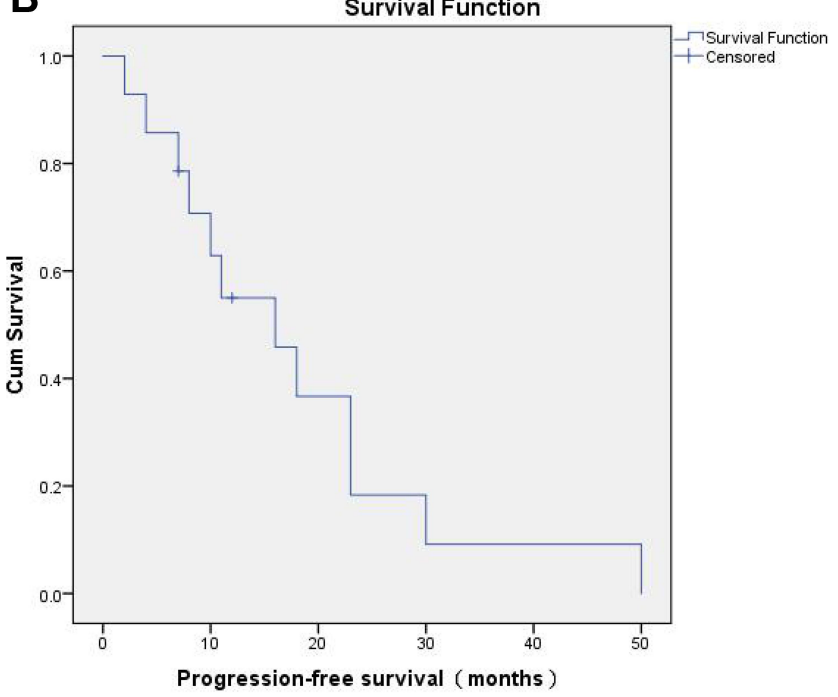

Figure 4 (A) Overall survival of all patients enrolled in our study. (B) Progression-free survival of all patients enrolled in our study. 

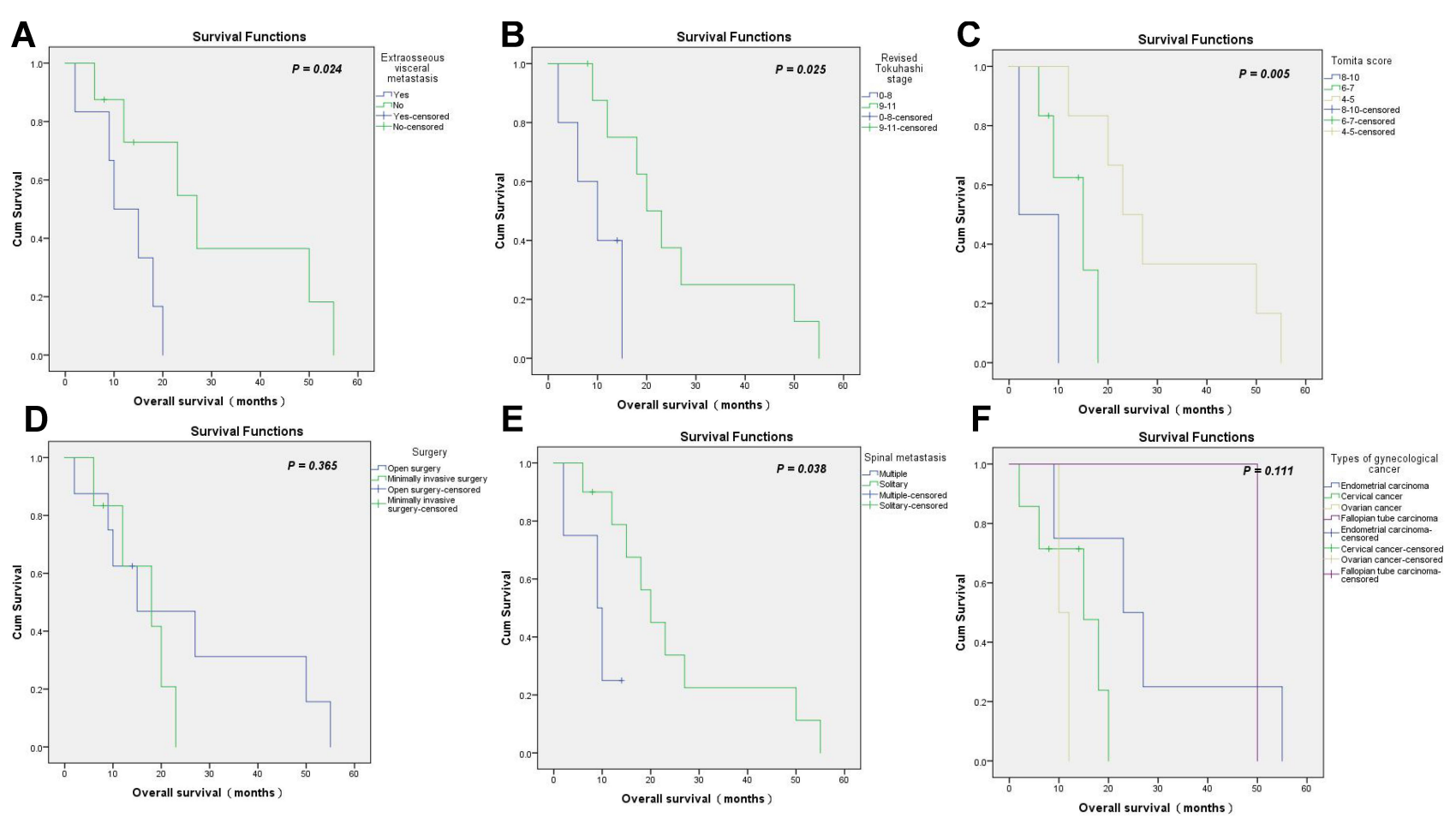

Figure 5 Univariate analysis of prognostic factors affecting overall survival. $(\mathbf{A})$ Extraosseous visceral metastasis $(p=0.024) .(\mathbf{B})$ Revised Tokuhashi stage $(P=0.025)$. $(\mathbf{C})$ Tomita stage $(p=0.005)$. (D) Surgery $(p=0.365)$. $(E)$ Number of spinal lesions $(p=0.038)$. $(\mathbf{F})$ Types of gynecological cancer $(P=0.1 \mathrm{I} I)$.
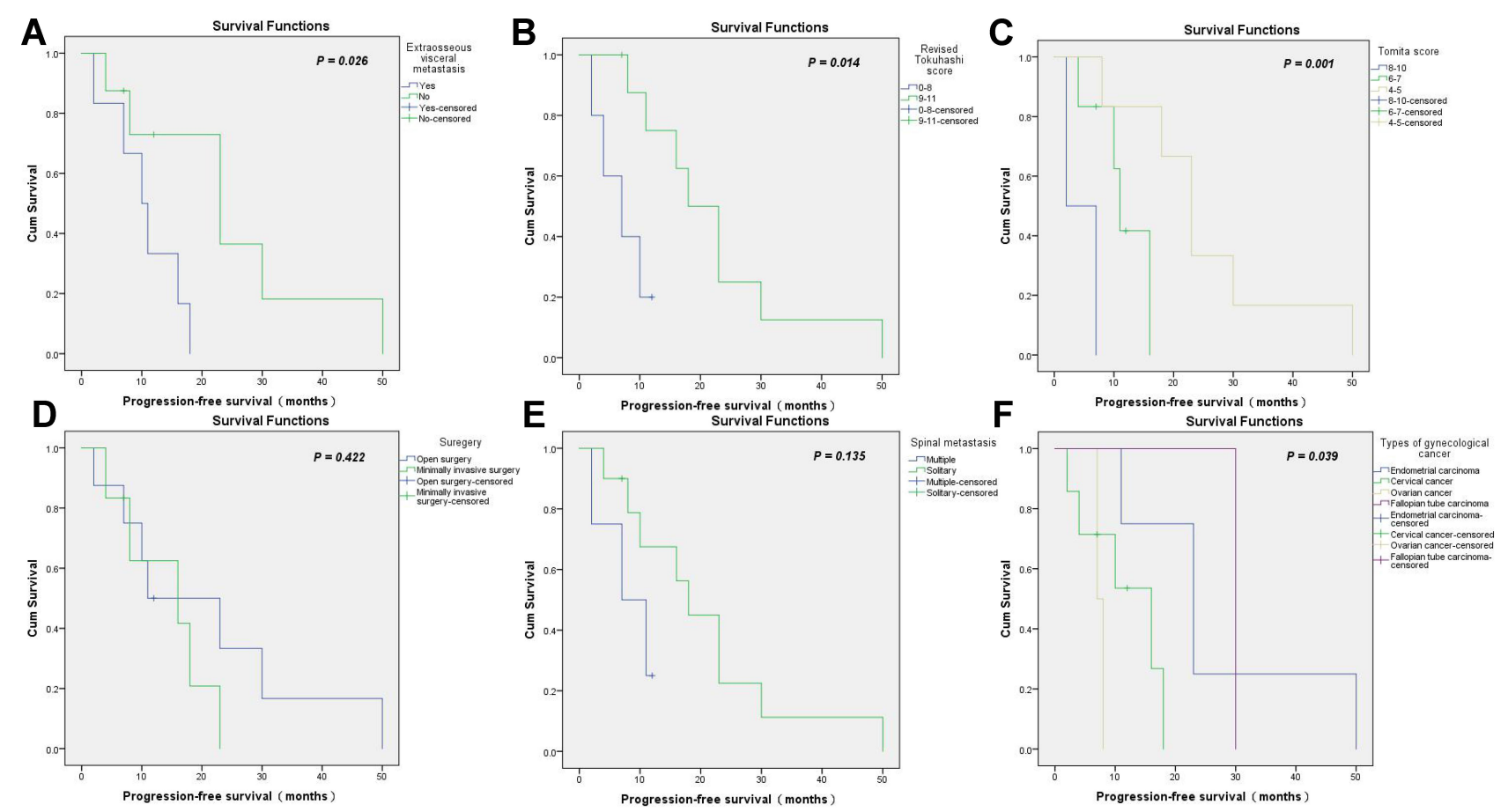

Figure 6 Univariate analysis of prognostic factors affecting progression-free survival. $(\mathbf{A})$ Extraosseous visceral metastasis $(p=0.026)$. $(\mathbf{B})$ Revised Tokuhashi stage $(p=$ 0.014). (C) Tomita stage $(p=0.00 I)$. (D) Surgery $(p=0.422)$. (E) Number of spinal lesions $(p=0.135)$. (F) Types of gynecological cancer $(p=0.039)$.

posterior tumor resection, spinal cord decompression, and titanium rod system spinal reconstruction. The average blood loss during operation was $650 \mathrm{~mL}$ (range 100-
1200) $\mathrm{mL}$. Six patients underwent minimally invasive bone cement percutaneous vertebroplasty, with an average blood loss of $35 \mathrm{~mL}$ (range 20-30) during the operation. 
No clear complications occurred during the perioperative period, and the original treatment plan was continued after the operation. Therefore, we advocate that in patients with neurological deficits caused by metastatic spinal cord compression, open surgery should be performed in a more thorough manner to remove the spinal metastases. For patients without spinal cord compression, percutaneous vertebroplasty can be recommended.

At present, the treatment of patients with MSGC is mainly to relieve symptoms. ${ }^{20-22}$ The purpose of treatment is to relieve pain, prevent the occurrence of pathological fractures, prevent the progression of the disease, improve function, and prolong survival time. Treatment options include comprehensive antitumor therapy (surgery + radiotherapy + chemotherapy) and bone resorption suppression therapy (bisphosphonate therapy). ${ }^{20-26}$ Chemotherapy and radiotherapy have been shown to benefit patients with bone metastases from cervical and ovarian cancer. ${ }^{20-26}$ Bisphosphonates have been shown to be effective in preventing bone-related events and alleviating the symptoms of bone pain in patients with bone metastases. ${ }^{27}$ Some scholars believe that simultaneous radiotherapy and chemotherapy can significantly reduce the occurrence of distant metastasis and local lesion progression of cervical cancer cells compared with simple radiotherapy, which may improve quality of life and prolong survival. In our study, 14 patients with MSGC received chemotherapy, and 9 patients received radiation therapy.

The OS after spinal surgery was 19.2 months (range 2-55), and patients underwent an average of 19.2 months (range 255 ) of postoperative follow-up. The 1-year and 2-year survival rate for all patients was $64.3 \%$ and $21.4 \%$, respectively. Compared to Liu and Zhong's reports, longer survival time was observed in our case series. There may be two reasons for this conclusion; our study adhered to more stringent indications for surgical intervention and patients with advanced disease were not included. In addition, their study included low patient numbers so it is difficult to draw reliable conclusions. The selection bias inherent in rare and inconsistent retrospective studies can severely affect the statistical results from separate study series.

Our univariate analysis showed that extraosseous visceral metastasis $(p=0.024)$, revised Tokuhashi stage $(p=0.025)$, Tomita stage $(\mathrm{p}=0.005)$, and number of spinal lesions $(\mathrm{p}=$ 0.038 ) were related to OS. Extraosseous visceral metastasis ( $p$ $=0.026)$, revised Tokuhashi stage $(\mathrm{p}=0.014)$, Tomita stage $(\mathrm{p}$ $=0.001)$, and gynecological cancer type $(\mathrm{p}=0.039)$ were also associated with PFS. No significant differences were found among other factors. Extraosseous visceral metastasis was also associated with OS $(p=0.043)$ and PFS $(p=0.044)$ in patients with MSGC.

The revised Tokuhashi stage and Tomita stage correspond to the general condition and nerve function of the patient. ${ }^{28,29}$ Patients with good overall health can withstand surgery and subsequent adjuvant therapy. Conversely, poor general condition and impaired nervous system function mean that surgical treatment may pose a risk to the patient. Visceral metastasis is an important prognostic factor in patients with metastatic spinal tumors. Multiple bone involvement and extraosseous metastasis are also considered to be prognostic factors for bone metastases in cervical cancer and endometrial cancer. ${ }^{3-7,30}$ In our series of studies, the number of spinal metastases were closely related to OS in univariate analysis, while the factor did not show significant correlation with PFS. The root cause may be that bone metastasis itself may not directly cause death. On the contrary, visceral metastasis destroys the physiological function of the main internal organs and should be regarded as a fatal factor. The number of spinal metastases overall is also an important concern. In addition, for patients with no visceral metastasis and a small number of spinal metastases, more aggressive surgical treatment may be beneficial to better control spinal lesions, which is also consistent with Tomita and revised Tokuhashi scores.

We found the type of gynecologic cancer to be an potential prognostic factor for the PFS of patients with MSGC, and survival according to tumor type is highest for endometrial cancer, followed by cervical cancer and ovarian cancer. The above conclusion is also consistent with results reported in the literature. $^{3-6,30}$ Importantly, there are other subtypes of gynecological cancer such as fallopian tube cancer and endometrial stromal sarcoma that were hardly included in our study; therefore, further research is needed to clarify the clinical features of MSGC and associated prognosis.

However, this study has several limitations. First of all, it is a retrospective analysis, limited by the number of cases. A more accurate and comprehensive statistical analysis cannot be carried out, and it is impossible to identify the specific prognostic factors. Furthermore, this study included only with MSGC patients who underwent spinal surgery but did not include patients who did not undergo spinal surgery. Naturally, this will bring a certain bias in the selection of the results. Since this is a retrospective analysis that lasted over ten years, we could not study the molecular characteristics of tumors during diagnosis and treatment. Besides, a variety of treatment methods for gynecological tumor bone metastasis have developed rapidly over the past ten years, which is another limitation of this study. Despite these limitations, 
however, this study is one of the most extensive single-center retrospective studies in Asia, focusing on the surgical treatment of MSGC. It took about ten years of more than 1000 patients with spinal metastases at our institution. Thus, it has clinical significance.

In short, spinal metastases in patients with GC are very rare, and MSGC patients have significantly improved quality of life after surgical treatment. The results of this study indicate that revised Tokuhashi stage, Tomita stage, subtypes of gynecological cancer, and the number of spinal lesions are potential prognostic factors for the survival of patients with MSGC. However, due to the limited number of patients and short follow-up time, our understanding of MSGC is still limited. Importantly, the surgical treatment and adjuvant treatment adopted in this study are more standardized than previous reports, and the follow-up results are true and reliable. We look forward to future prospective and large-scale research on this challenging issue, which we hope will improve quality of life and extend survival time in patients with MSGC.

\section{Data Sharing Statement}

The anonymized data used and/or analyzed during the current study are available from the corresponding authors on reasonable request.

\section{Ethics Approval and Consent to Participate}

This study was conducted with approval from the Ethics Committee of Peking Union Medical College Hospital. This study was conducted in accordance with the declaration of Helsinki. All patients have provided written informed consent for the case details to be published and all patients provided informed consent to have their medical records reviewed for this study.

\section{Consent for Publication}

Consent for publication was obtained from each patient.

\section{Acknowledgments}

We would like to thank our colleagues at the Departments of Obstetrics and Gynecology, Anesthesiology, Pathology, Nuclear medicine, Radiology, and Orthopaedic surgery for their support.

\section{Author Contributions}

All authors made a significant contribution to the work reported, whether that is in the conception, study design, execution, acquisition of data, analysis and interpretation, or in all these areas; took part in drafting, revising or critically reviewing the article; gave final approval of the version to be published; have agreed on the journal to which the article has been submitted; and agree to be accountable for all aspects of the work.

\section{Funding}

This study was supported by Peking Union Medical College Graduate Student Innovation Fund (2018) (Project No. 2018-1002-02-08; Grant recipient: S.L.). The funder had no role in study design, data collection and analysis, decision to publish, or preparation of the manuscript.

\section{Disclosure}

The authors report no conflicts of interest for this work.

\section{References}

1. Chen W, Zheng R, Baade PD, et al. Cancer statistics in China, 2015. CA Cancer J Clin. 2016;66(2):115-132. doi:10.3322/caac.21338

2. Siegel RL, Miller KD, Jemal A. Cancer Statistics, 2017. CA Cancer J Clin. 2017;67(1):7-30. doi:10.3322/caac.21387

3. Gao X, Zhao C, He S, et al. Treatment and outcomes of 28 patients with spinal metastasis from gynecological cancer. $J$ Neurooncol. 2018;137(2):387-394. doi:10.1007/s11060-017-2728-x

4. Kocaer M, Gulseren V, Ozdemir IA, et al. Management of vertebral metastasis in patients with uterine cervical cancer. Int J Gynecol Cancer. 2018;28(6):1191-1195. doi:10.1097/IGC.0000000000001273

5. Qin DA, Song JF, Song LP, et al. Integrated approach to pain management for a patient with multiple bone metastases of uterine cervical cancer. J Int Med Res. 2018;46(5):2023-2030. doi:10.1177/ 0300060518763709

6. Yoon A, Choi $\mathrm{CH}$, Kim HJ, et al. Contributing factors for bone metastasis in uterine cervical cancer. Int J Gynecol Cancer. 2013;23 (7):1311-1317. doi:10.1097/IGC.0b013e31829da127

7. Makino H, Nishio S, Tsubamoto H, et al. Treatment and prognosis of bone metastasis from cervical cancer (KCOG-G1202s). J Obstet Gynaecol Res. 2016;42(6):701-706. doi:10.1111/jog.12956

8. Kehoe SM, Zivanovic O, Ferguson SE, et al. Clinicopathologic features of bone metastases and outcomes in patients with primary endometrial cancer. Gynecol Oncol. 2010;117(2):229-233. doi:10.1016/j.ygyno.2010.01.047

9. Uccella S, Morris JM, Bakkum-Gamez JN, et al. Bone metastases in endometrial cancer: report on 19 patients and review of the medical literature. Gynecol Oncol. 2013;130(3):474-482. doi:10.1016/j. ygyno.2013.05.010

10. Zhang M, Sun J. Bone metastasis from ovarian cancer. Clinical Analysis of 26 Cases . Saudi Med J. 2013;34(12):1270-1273.

11. Liu A, Sankey EW, Goodwin CR, et al. Postoperative survival and functional outcomes for patients with metastatic gynecological cancer to the spine: case series and review of the literature. $J$ Neurosurg Spine. 2016;24(1):131-144. doi:10.3171/2015.3.SPINE15145

12. Thanapprapasr D, Nartthanarung A, Likittanasombut $\mathrm{P}$, et al. Bone metastasis in cervical cancer patients over a 10-year period. Int $J$ Gynecol Cancer. 2010;20(3):373-378. doi:10.1111/ IGC.0b013e3181d4a0a1 
13. Miranpuri AS, Rajpal S, Salamat MS, et al. Upper cervical intramedullary spinal metastasis of ovarian carcinoma: a case report and review of the literature. J Med Case Rep 2011; 5: 311.

14. Rades D, Schild SE, Dunst J. Radiotherapy is effective for metastatic spinal cord compression in patients with epithelial ovarian cancer. Int $J$ Gynecol Cancer. 2007;17(1):263-265. doi:10.1111/j.15251438.2006.00765.x

15. Li H, Wu X, Cheng X. Advances in diagnosis and treatment of metastatic cervical cancer. J Gynecol Oncol. 2016;27(4):e43. doi:10.3802/jgo.2016.27.e43

16. Zhang Y, Guo X, Wang G, et al. Real-world study of the incidence, risk factors, and prognostic factors associated with bone metastases in women with uterine cervical cancer using Surveillance, Epidemiology, and End Results (SEER) data analysis. Med Sci Monit. 2018;24:6387-6397. doi:10.12659/MSM.912071

17. Tiwari A, Kumar N, Bajpai R, et al. Bone metastasis from ovarian cancer. J Cancer Res Ther. 2007;3(1):34-36. doi:10.4103/0973-1482.31969

18. Bakkum-Gamez JN, Gonzalez-Bosquet J, Laack NN, et al. Current issues in the management of endometrial cancer. Mayo Clin Proc. 2008;83(1):97-112. doi:10.4065/83.1.97

19. Abdul-Karim FW, Kida M, Wentz WB, et al. Bone metastasis from gynecologic carcinomas: a clinicopathologic study. Gynecol Oncol. 1990;39(2):108-114. doi:10.1016/0090-8258(90)90414-G

20. Huang J, Lei D. Intramedullary spinal cord metastasis from ovarian cancer in a 50-year-old female. World Neurosurg. 2017;106:1049.e31049.e4. doi:10.1016/j.wneu.2017.07.040

21. Safadi S, Rendon P, Rutledge $\mathrm{T}$, et al. Ovarian carcinoma with isolated spinal cord metastasis. J Investig Med High Impact Case Rep. 2016;4(3):2324709616657644.
22. Adachi H, Hanakita J, Takahashi T, et al. Precocious solitary cervical metastasis from endometrial cancer presenting as cervical radicular pain. World Neurosurg. 2018;118:162-167. doi:10.1016/j.wneu.2018.07.042

23. Eskander JP, Kuris EO, Younghein AJ, et al. Spinal metastases from a primary fallopian tube serous adenocarcinoma: a case report. Global Spine J. 2015;5(5):e65-8. doi:10.1055/s-0035-1546417

24. Batista LM, Carvalho CH, Acioly MA, et al. Spinal metastasis of endometrial stromal sarcoma: clinicopathological features and management. Surg Oncol. 2011;20(2):e78-83. doi:10.1016/j.suronc.2010.10.006

25. Cormio G, Colamaria A, Di Vagno G, et al. Surgical decompression and radiation therapy in epidural metastasis from cervical cancer. Eur J Obstet Gynecol Reprod Biol. 2000;89(1):59-61. doi:10.1016/ S0301-2115(99)00165-7

26. Takeshita S, Todo Y, Okamoto K, et al. Thoracic laminectomy with spinal fixation in a nonambulatory patient with metastatic vertebral tumor from endometrial carcinoma. J Obstet Gynaecol Res. 2016;42 (10):1395-1399. doi:10.1111/jog.13054

27. Coleman RE, McCloskey EV. Bisphosphonates in oncology. Bone. 2011;49(1):71-76. doi:10.1016/j.bone.2011.02.003

28. Tokuhashi $\mathrm{Y}$, Matsuzaki $\mathrm{H}$, Oda $\mathrm{H}$, et al. A revised scoring system for preoperative evaluation of metastatic spine tumor prognosis. Spine (Phila Pa 1976). 2005;30(19):2186-2191. doi:10.1097/01. brs.0000180401.06919.a5

29. Tomita K, Kawahara N, Kobayashi T, et al. Surgical strategy for spinal metastases. Spine (Phila Pa 1976). 2001;26(3):298-306. doi:10.1097/00007632-200102010-00016

30. Arrigo RT, Kalanithi P, Cheng I, et al. Predictors of survival after surgical treatment of spinal metastasis. Neurosurgery. 2011;68(3):674-81; discussion 681. doi:10.1227/NEU.0b013e318207780c

\section{Publish your work in this journal}

Cancer Management and Research is an international, peer-reviewed open access journal focusing on cancer research and the optimal use of preventative and integrated treatment interventions to achieve improved outcomes, enhanced survival and quality of life for the cancer patient.
The manuscript management system is completely online and includes a very quick and fair peer-review system, which is all easy to use. Visit http://www.dovepress.com/testimonials.php to read real quotes from published authors. 